\title{
A Distributed Machine Learning Approach for the Secondary Voltage Control of an Islanded Micro-Grid
}

\author{
Miftah Al Karim, Jonathan Currie, Tek-Tjing Lie
}

\begin{abstract}
Balancing the active and the reactive power in a stand-alone micro-grid is a critical task. A micro-grid without energy storage capability is even more vulnerable to stability issues. This paper investigates a distributed secondary control to maintain the rated voltage in a stand-alone micro-grid. Here multiple machine learning algorithms have been implemented to provide the secondary control where a primary control scheme is insufficient to maintain a stable voltage after a sudden change in the load. The performance of the secondary control is monitored by a centralized system and in most of the cases it does not interfere. Based on different contingencies the proposed method would suggest different machine learning algorithms which are previously trained with similar data. The contingencies are based on an imbalance either in the active or in the reactive power in the system. It is considered that the distributed generators such as the wind and solar plants as well as the residential loads have some degree of randomness. The secondary control is invoked only in the events when primary droop control is insufficient to address the stability issue and maintain a desired voltage in the system.
\end{abstract}

Index Terms-Neural network, automatic generation control, load forecasting, distributed generation, wind energy, solar energy.

\section{INTRODUCTION}

The concept of a smart grid emerged to improve reliability and sustainability through intelligent means. The idea is to offer better strategies for the operation that conventional electric power system often struggle to realize. Distributed energy generation, deregulated energy market, government incentives and demand response are some of the key attributes that demand an enhanced control architecture in a modern power system. Recent studies show that the micro-grids can be considered as the building blocks of a smart grid due to the distributed and stochastic characteristics that allow bi-directional energy flow [1], [2]. A micro-grid is a smaller, distributed and somewhat independent entity in a power system that mostly provides energy to a remote distribution network. It usually contains distributed energy generation facilities such as wind power generation, solar power generation, gas turbine based plants, diesel generator based plants, fuel cells, energy storage devices etc. Generally, a micro-grid strives to mitigate local demand from its own inventory. If required, it can borrow or provide power to the grid [3]. Micro-grids can also be operated in stand-alone basis. Usually, a stand alone micro-grid appears out of a fault or it is built in a remote location or island.

The voltage and frequency in a micro-grid are generally controlled by synchronous generators. Due to the stochastic or un- certain nature of solar and wind power generation, power electronic interfaces such as inverters and converters are often used in a micro-grid. These uncertainties can influence innovative strategies for droop control [4]. As electricity production must be balanced with the consumption, the random nature of the distributed generators often pose threats against maintaining this balance. This characteristic sometimes requires the microgrids to deploy energy storage mechanism [5]. On the other hand operation of a micro-grid without any energy storage device has also been considered due its cost effectiveness and high power quality under normal operation scenarios. However operating a micro-grid without an energy storage poses many challenges [6]. In [7] an energy storage less control scheme that utilizes pulse width modulation technique for maximum power tracking from PV-array and fuzzy logic based diesels generator speed control is proposed. Such mechanism is good enough for the PV-diesel based hybrid system, however, the diesel generator used in the article had a maximum capacity higher than the peak load predicted to provide full energy support at night. This planning strategy would not be sufficient for a wind-PV based isolated micro-grid that uses synchronous generators having lower capacity than the peak demand.

Micro-grids with sufficient distributed energy generation capacity may use low capacity synchronous generators [8], but in those micro-grids during an emergency situation optimized load shedding may be a necessity [9]. A typical micro-grid often does not accommodate the concept of having a base load as large variations may be expected due to the frequent use of inductive loads [10]. Due to this reason, optimization techniques are often discussed in the literature to manage the controllable resources [11]-[13]. Commands, based on those optimization techniques can be carried out from a control center, however, the continuous bi-directional communication could be an expensive venture [14]. To mitigate these issues, research such as [15] proposed decentralized control schemes at every subsystem node, but how the uncertainty of the distributed energy resources influences the control schemes in each of the sections have not been adequately discussed, which is one of the key focuses in any decentralized microgrid management system [16].

Decentralized control schemes such as droop controller is one of the most popular schemes. Yet, this scheme, time to time may suffer from the presence of the small signal instability issues [17]. This paper proposes a distributed machine learning algorithm architecture, established on the 
synchronous generators in order to provide a secondary control scheme under different scenarios. The underlying purpose of the secondary control is to maintain the desired voltage level throughout a micro-grid by identifying and mitigating an imminent voltage instability problem. The designed microgrid would be in a stand-alone mode without having access to an energy storage device. The machine learning algorithms would be trained based on the historical energy production data and the variable demand data.

\section{MICRO-GRID MODEL}

This paper follows the lumped load on a common transmission grid approach shown in [13], [15], [18]. However, this model differs in dividing the system load into two parts critical and controllable loads [19]. The critical loads are comparable to the base load of a system that remains unchanged throughout a certain period. While the controllable loads are unpredictable in nature and sudden changes can be observed at any given time. The system has two thermal power plant run by synchronous generators, a wind farm, and a solar plant. The overall demand in the system is higher than the total capacity of the synchronous generators. This means the wind power plant and the solar plant play a significant role in mitigating the demand. The wind power plant deploys an induction generatorbased variable speed wind turbine. While the solar power plant is designed as a current source with a diode, multiple resistances (internal and leakage current) followed by a voltage source converter (VSC) [20]. Voltage source converters with appropriate controllers can ensure grid stability, however in this paper, a VSC based solar plant is only implemented as a variable energy source. Both the solar plant and the wind power plant are not subject to the secondary voltage and frequency control rather from the secondary controller's point of view these plants serve as a data source for the proposed binary classifier using a decision tree based machine learning algorithm.

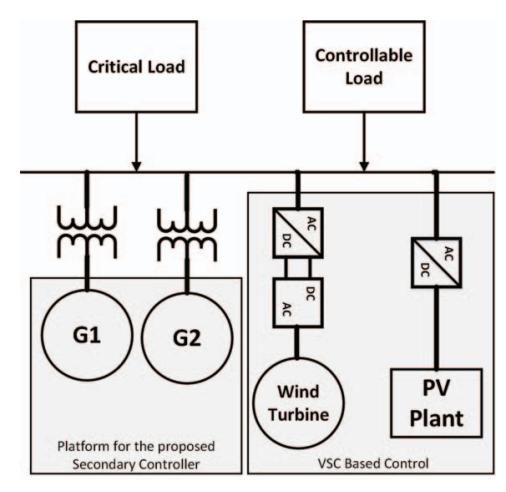

Figure 1. The Micro-grid Model a modified approach from [21]

\section{Proposed SECONDARY CONTROL}

The purpose of the primary control which is a part of the hierarchical control scheme in a power system, is mainly to stabilize the system frequency and voltage.The primary control in a standalone micro-grid that does not have any storage units may cause frequency deviation even if the system remains at a steady state condition. The secondary control acts on the deviations observed after the primary control has been implemented and tries to balance the system. The secondary control has a slower dynamics than the primary control in a power system, the idea behind that is to introduce a decoupling mechanism between these two [21]. Although the usual practice is to implement the secondary control centrally in this paper a distributed supervised secondary control scheme is proposed. The initial feedback data to trigger the secondary control is taken from the transmission line voltage and the frequency of the individual machine. The secondary control is applied after the machine initialization has been taken care of and the system has reached to steady state condition. The proposed secondary control acts as a restorative measure over the primary controller. This paper follows a similar approach for the conventional droop characteristics principle discussed in [21] for the primary frequency and voltage control. This principle observes that the droop control coefficients for active and reactive power $D_{P}$ and $D_{Q}$ should be determined based on the nominal ratings of the individual machine. By calculating $D_{Q i} Q_{N i}$ and $D_{P i} P_{N i}$ and subtracting those from the no-load output voltage and angular frequency, the reference voltage and angular frequency can be fine-tuned during standalone operation of the micro-grid. The overall distribution should satisfy the condition $D_{P i} P_{N i}=\Delta \omega_{\max }$ and $D_{Q i} Q_{N i}=$ $\Delta E_{\max }$. Where, ' $\mathrm{N}$ ' is the number of distributed energy generators which in this paper is two and ' $\mathrm{P}$ ' and ' $\mathrm{Q}$ ' are the generated active and reactive power, $\Delta \omega_{\max }$ and $\Delta E_{\max }$ are the maximum allowable angular frequency and voltage deviations. After that the dynamic response can be observed through linearizing the active and reactive power equations to find out their small signal models.

$$
\begin{aligned}
& \Delta P(s)=\frac{G}{s+D_{P} G} \Delta \omega^{*}(s) \\
& \Delta Q(s)=\frac{H}{1+D_{Q} H} \Delta E^{*}(s)
\end{aligned}
$$

Here, $G$ and $H$ depend on the nominal terminal voltage and load angle of the generator and the transmission line voltage where power is delivered. Through the deviation in angular frequency, the primary controller controls the active power reference to the synchronous generators that inturn controls the active power generation and through the deviation in terminal voltage the controller controls reactive power generation from the synchronous generators. This paper implements the secondary control scheme once the threshold of the $\Delta \omega_{\max }$ and $\Delta E_{\max }$ have been breached under a steady state operating condition. Figure-2 shows the model of the secondary control system. The secondary control is considered as a part of the hierarchical control of the micro-grid [4], [21], [22]. In this paper, the secondary control is designed based on the distributed machine learning algorithms trained under different 
voltage stability conditions. The following section discusses the preparation of the control algorithm. The main objective of designing the algorithm is to maintain the voltage quality at the transmission line [21], [22].

\section{A. Data Preparation}

The proposed machine learning based secondary control scheme is applied to the synchronous machines based on the amount of power generation available from the wind and solar power plant as well as the controllable residential loads. The algorithm works in a cascaded form. The initial part of the algorithm is a binary classification prepared with four different attributes and one target attribute. The available wind power, the available solar power, controllable loads and the sudden changes in load are the input attributes. The target attribute is the binary class of the system either being stable or unstable. For establishing such a relation in this study, the data set has been prepared out of fifty randomly selected scenarios chosen at a very close to voltage instability point to intentionally reproduce voltage instability at times. Thus, the operating point of the system is chosen near the lowest voltage margin on the system PV-curve [23] shown in Figure-3.

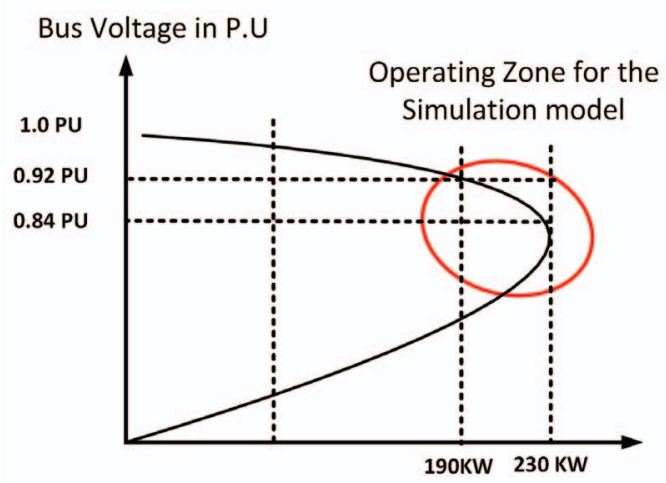

Active power Transmitted to controllable loads (Without Compensation)

Figure 3. Operating Near the Voltage Instability Point (Without Compensation) [23]

As the system has been forced to operate near its stability margin, the probability of losing the system stability increases with a sudden change in load. The simulation model ensures a significant change in load to introduce voltage instability in the system. The sudden change in load breaches the active power margin that introduces power swing in the system specially between the two synchronous generators due to the impact of primary controller in both the locations. To observe the instability of the system, fifty random incidents have been prepared. It is shown in Table-I.

The transmission line voltage is then observed of this fifty incidents. The voltage characteristics are distinguished based on its difference $\Delta V$ between any two points $N$ and $N-1$. The

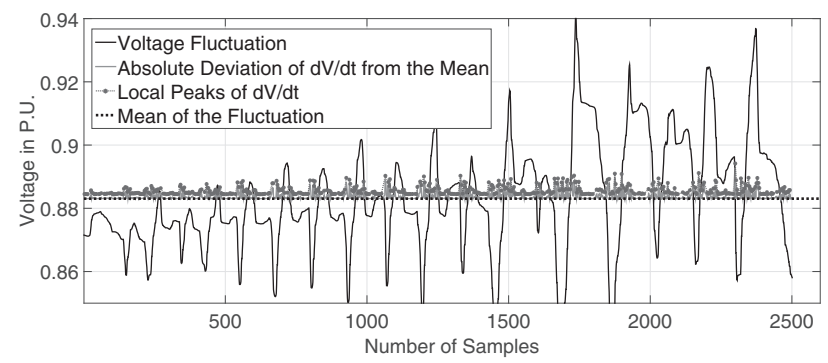

Figure 4. Fluctuation During Voltage Instability

characteristics of the transmission line voltage are monitored using a four-second window. Within this four second window if the voltage fluctuation crosses the allowable fluctuationthreshold $\frac{\Delta V_{\max }}{\Delta T}$ more than a certain predefined number $M$, then the voltage fluctuation is considered as persisting and the individual scenario is labeled as unstable. Otherwise, the voltage of the system is considered as stable. A similar approach is also proposed for the rotor angular frequency at the individual synchronous generator.

\section{B. Preparing the Distributed Algorithm}

Once the model data has been prepared, an unsupervised K-means cluster has been prepared. The purpose of using an unsupervised cluster is to narrow down the target data before preparing another machine learning algorithm, the neural network. Unsupervised clustering would make sure that the data falls under one category at a time. Five predefined categories have been chosen for the clusters. The purpose of using the neural network is to do parameter fitting. In this case, the parameters are the references for rotor speeds and the references for field voltages of the two synchronous generators. For a better prediction accuracy, in this paper separate neural networks are proposed for five different clusters [24]. The clustering data is shown in the Table-I. The clustering process is shown in Figure-5.

Table I

Data TABle With Clusters (Units in Watts)

$\begin{array}{ccccc}\text { Wind Power } & \text { Solar Power } & \text { Residential Load } & \text { Load Change } & \text { Cluster } \\ 200000 & 200000 & 203000 & 28000 & 4 \\ 187500 & 200000 & 204000 & 50000 & 4 \\ 225000 & 100000 & 198000 & 50000 & 5 \\ 187500 & 150000 & 198000 & 47000 & 1 \\ \ldots & \ldots & \ldots & \ldots & \ldots \\ 187500 & 100000 & 204000 & 47000 & 5 \\ 225000 & 100000 & 191000 & 45000 & 5 \\ 200000 & 50000 & 192000 & 42000 & 3 \\ 187500 & 100000 & 201000 & 46000 & 5\end{array}$

The second step of the algorithm is to prepare a classification routine using an ensemble of bagged decision trees. The purpose of this classifier is to establish a binary class of stability. Some of the aforementioned fifty incidents are stable and some are unstable. The bagged decision tree classifier is trained with these incidents for future predictions. Once a new set of data arrives the binary classifier is invoked to 


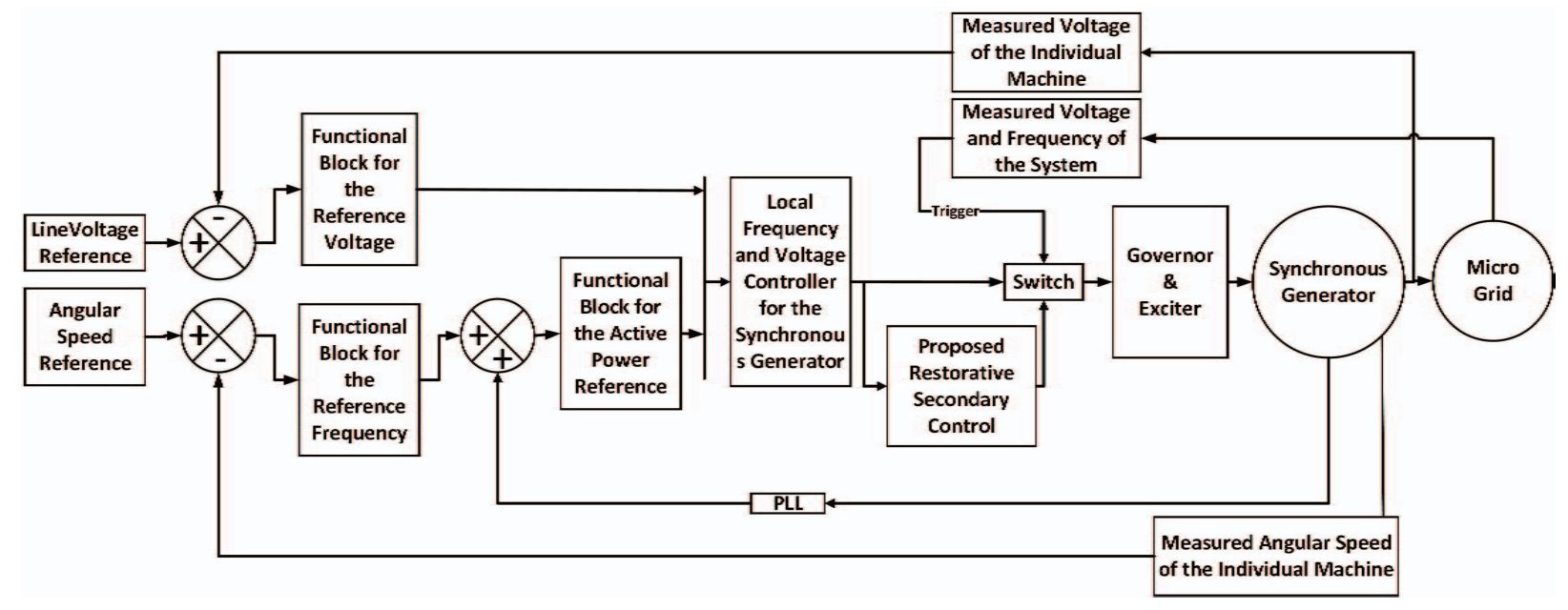

Figure 2. Secondary Control Scheme

predict the imminent outcome of this incident during a sudden and significant load change. If the classifier predicts probable instability, based on the previously prepared cluster value of that particular incident a suitable neural network is selected. The purpose of the neural network in the individual cluster is to propose necessary modifications in the active and reactive power control that is currently under operation through a primary controller. The modifications are applied based on the scenarios if the systems were stable with a similar amount of remaining residential load. It means the neural network under each cluster is prepared based on the final value of the residential load ('post-event') considering that the sudden change has occurred. The system is simulated with the 'Postevent' load scenario maintaining a steady state condition. The output of the neural network predicts the appropriate value of the reference rotor speed $\omega_{S G}$ as well as reference field voltage $V_{f l d}$ of the individual synchronous generator (SG) at the steady state condition. The purpose of the neural network in this paper is to calibrate the primary control system for the aforementioned fifty predefined conditions. The overall architecture of the algorithm is shown in Figure-6.

\section{RESUlts AND Discussions}

The test micro-grid showed in Figure-1 has been used to apply the fifty different scenarios close to the system instability points. Then the decision tree applied and tested on the system data and a binary classifier has been prepared. The result obtained from the classifier is shown in Figure-7.

In a parallel working stream, the data is also fed to an unsupervised clustering algorithm and five different data clusters have been prepared. The centroids of each cluster have been stored for the purpose of labeling the newly arrived data. The data labeling is based on the previously prepared clusters. The centroids of each cluster are given in the Table-II.

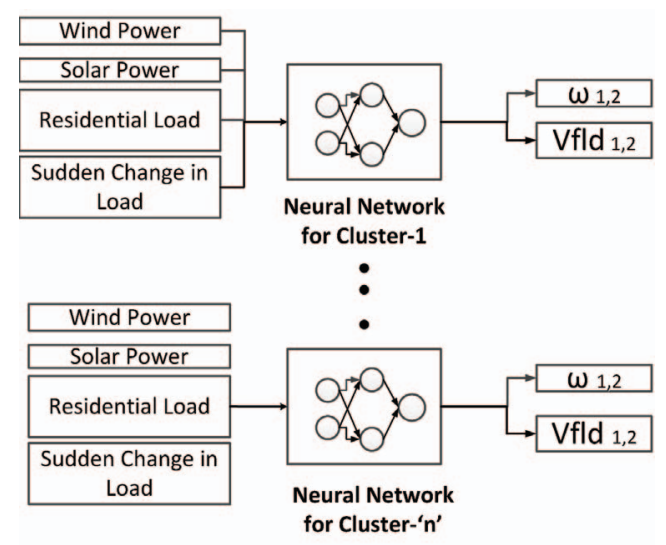

Figure 5. K-means Cluster based Neural Networks [24]

Table II

Centroids of THE Clusters

$\begin{array}{ccccc}\text { Cluster } & \text { Wind(kW) } & \text { Solar(kW) } & \text { Load }(\mathrm{kW}) & \text { Changes }(\mathrm{kW}) \\ 1 & 191 & 150 & 198 & 42 \\ 2 & 190 & 200 & 202 & 36 \\ 3 & 204 & 50 & 200 & 39 \\ 4 & 200 & 100 & 202 & 44 \\ 5 & 198 & 0 & 199 & 44\end{array}$

When a new system data has been collected and the decision tree based classifier predicts a probable voltage instability in the system. Then based on the proposed clustering scheme an appropriate neural network has been invoked and prepared for the imminent threat. If the system identifies voltage instability the secondary control scheme forces active and reactive power control on each of the generators based on the predicted outcomes of the neural networks. The result of the two neural networks are shown in Table-III. 


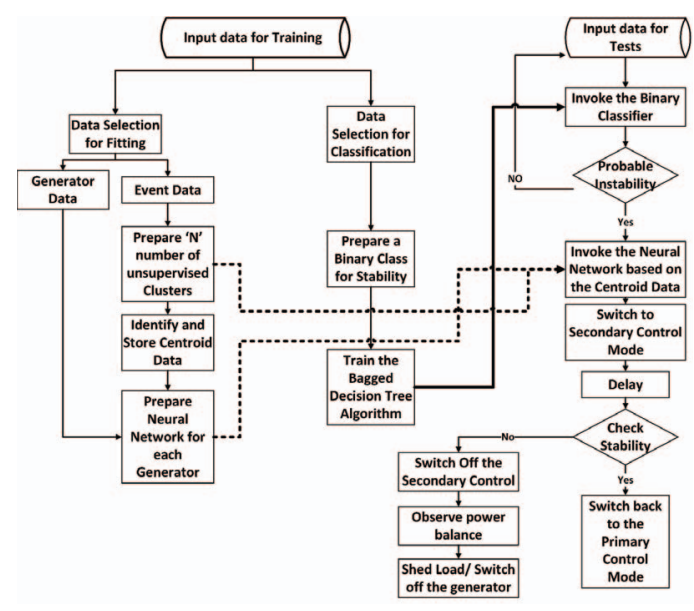

Figure 6. Work-Flow of the proposed Algorithm

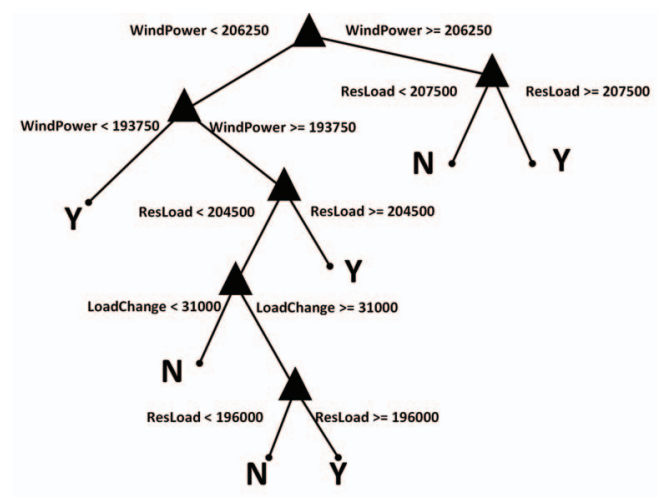

Figure 7. Binary Classification of the System Stability (Units in watts)

Table III

Predictions Using NeURal Networks

Active Power Reference: Generator-1

\begin{tabular}{cccc}
\hline Cluster & Actual & Neural Net & Error \% \\
\hline 4 & 0.8013 & 0.848 & 5.828 \\
3 & 0.87 & 0.849 & 2.41 \\
Field Voltage Reference: Generator-1 & & & \\
\hline Cluster & Actual & Neural Net & Error \% \\
\hline 4 & 1.00 & 1.054 & 5.4 \\
3 & 1.00 & 1.0075 & 0.75 \\
Active Power Reference: Generator-2 & & & \\
\hline Cluster & Actual & Neural Net & Error \% \\
\hline 4 & 0.8014 & 0.86 & 7.31 \\
3 & 0.87 & 0.8709 & 0.103 \\
Field Voltage Reference: Generator-2 & & & \\
\hline Cluster & Actual & Neural Net & Error \% \\
\hline 4 & 1.00 & 1.065 & 6.5 \\
3 & 1.00 & 1.007 & 0.7
\end{tabular}

Finally, Figure- 8 shows the performance of the proposed algorithm in detecting and subsiding the voltage instability with the data cluster number ' 4 ', and Figure-9 shows the performance with data cluster number ' 3 '.
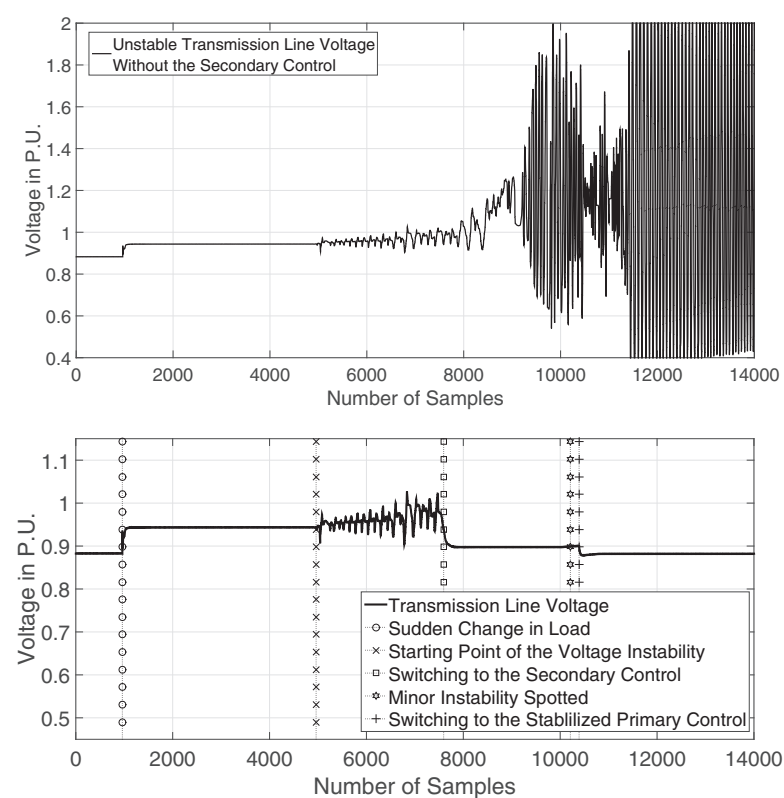

Figure 8. Performance of the Proposed Secondary Control Scheme (Cluster-4)
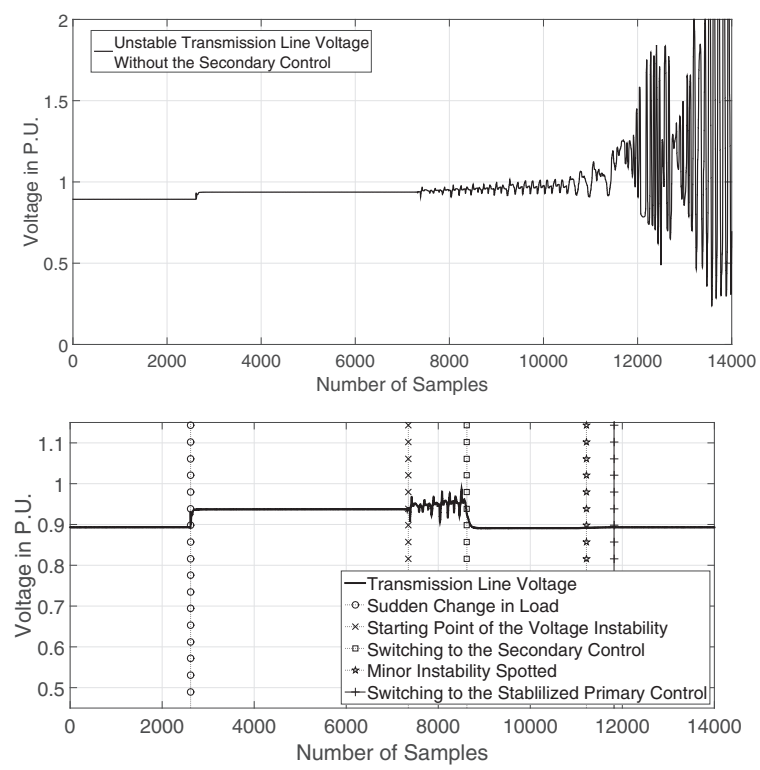

Figure 9. Performance of the Proposed Secondary Control Scheme (Cluster-3)

Figure- 8 and Figure-9 have been divided into multiple segments for better visualization. The circled lines on the left show the point where the system observes a sudden unexpected change in load. As the initial control scheme is the linearized primary droop control the immediate impact is a sudden rise in voltage. The voltage rise is followed by voltage instability that primary controller cannot control due to the mismatch in active power in the system. This point is shown using the $2^{\text {nd }}$ line with cross marks. The oscillation starts to rise and crosses the permissible maximum 
$\Delta V$ for the transmission line. At this particular moment when the instability is spotted the system forces secondary control mechanism over the synchronous generator. This transition is marked using the squares. After the secondary control is applied the transmission voltage becomes momentarily stable and reaches towards a new equilibrium point. In Table-III the performance of neural network in predicting reference values are shown. The table shows that the prediction has some errors in it. The observation also shows the predictability of the proposed method is working better with the data cluster- 3 than the data cluster- 4 . The results are coherent with Figure- 8 and Figure-9, in explaining why a better response is observed in Figure-9. The reason of such difference can be traced back to the bin count of the clusters. In the aforementioned fifty cases, cluster-3 has occurred fourteen times while the cluster four has occurred nine times. Such a difference could influence the training process of the neural network. Despite having these small errors the proposed secondary controller damps the oscillation effectively. However, due to that minor prediction error after the system becomes stable the transmission voltage tries to reach towards a new dynamic stability. This phenomenon is shown using the $4^{\text {th }}$ line with pentagrams. At that point of new dynamic stability, the system is switched back to its primary control mechanism. This switching is shown using the $5^{\text {th }}$ line with plus marks. The system remains stable after that point. The secondary controls applied in both the machines do not use any communication channel between them. The centralized control scheme for supervising the performance of the secondary control is only used when secondary control is incapable of maintaining stability and the centralized control would either shed additional load or switch the second synchronous generator having lower energy ratings off.

\section{Conclusion}

This study implements a cascaded method of applying machine learning algorithms in a distributed architecture to provide secondary supervisory control on an isolated microgrid. The results obtain through this study are significant. However, there is a need to discuss the impact of misclassification on the system operation and modification of the distributed control approach based on that misclassification. The performance of the classifier as well as neural network fitting model heavily depends on the number of use cases. In this paper, only fifty scenarios have been used near the power voltage margin. The accuracy of both the binary classifier and neural network fitting models would definitely improve if a large number of scenarios would be incorporated. Apart from this minor shortcoming, the performance of the algorithm can be considered a promising one.

\section{REFERENCES}

[1] R. H. Lasseter, "Smart distribution: Coupled microgrids," Proceedings of the IEEE, vol. 99, pp. 1074-1082, June 2011

[2] G. A. Pagani and M. Aiello, "Towards decentralization: A topological investigation of the medium and low voltage grids," Smart Grid, IEEE Transactions on, vol. 2, no. 3, pp. 538-547, 2011.
[3] J. Yuan, J. Ma, L. Zhao, and H. Liu, "A real-time balancing market clearing model for grid-connected micro-grid including energy storage system," in Smart Electric Distribution Systems and Technologies (EDST), 2015 International Symposium on, pp. 32-36, Sept 2015.

[4] J. Lopes, C. Moreira, and A. Madureira, "Defining control strategies for microgrids islanded operation," Power Systems, IEEE Transactions on, vol. 21, no. 2, pp. 916-924, 2006.

[5] T. R. Mtshali, G. Coppez, S. Chowdhury, and S. P. Chowdhury, "Simulation and modelling of PV-wind-battery hybrid power system," in 2011 IEEE Power and Energy Society General Meeting, pp. 1-7, July 2011.

[6] N. Argaw, R. Foster, and A. Ellis, "Renewable energy for water pumping applications in rural villages," New Mexico State, United States, 2003.

[7] A. Elmitwally and M. Rashed, "Flexible operation strategy for an isolated pv-diesel microgrid without energy storage," IEEE Transactions on Energy Conversion, vol. 26, pp. 235-244, March 2011.

[8] S. Singh, A. K. Singh, and S. Chanana, "Operation and control of a hybrid photovoltaic-diesel-fuel cell system connected to micro-grid," in Power India Conference, 2012 IEEE Fifth, pp. 1-6, Dec 2012.

[9] Y. Y. Hong, M. C. Hsiao, Y. R. Chang, Y. D. Lee, and H. C. Huang, "Multiscenario underfrequency load shedding in a microgrid consisting of intermittent renewables," IEEE Transactions on Power Delivery, vol. 28, pp. 1610-1617, July 2013.

[10] M. Kohansal, G. B. Gharehpetian, M. Rahmatian, M. Abedi, and M. J. Sanjari, "The effect of load condition on stability in isolated microgrids," in 2012 Second Iranian Conference on Renewable Energy and Distributed Generation, pp. 28-32, March 2012.

[11] S. Xing, "Microgrid emergency control based on the stratified controllable load shedding optimization," in Sustainable Power Generation and Supply (SUPERGEN 2012), International Conference on, pp. 1-5, Sept 2012.

[12] A. Y. Saber and G. K. Venayagamoorthy, "Smart micro-grid optimization with controllable loads using particle swarm optimization," in 2013 IEEE Power Energy Society General Meeting, pp. 1-5, July 2013.

[13] J. Xu, S. Tan, and S. K. Panda, "Optimization of economic load dispatch for a microgrid using evolutionary computation," in IECON 2011 - 37th Annual Conference on IEEE Industrial Electronics Society, pp. 3192 3197, Nov 2011.

[14] F. Katiraei, R. Iravani, N. Hatziargyriou, and A. Dimeas, "Microgrids management," Power and Energy Magazine, IEEE, vol. 6, no. 3, pp. 54 65, 2008.

[15] M. Babazadeh and H. Karimi, "Robust decentralized control for islanded operation of a microgrid," in 2011 IEEE Power and Energy Society General Meeting, pp. 1-8, July 2011.

[16] C. Wu, H. Mohsenian-Rad, J. Huang, and A. Y. Wang, "Demand side management for wind power integration in microgrid using dynamic potential game theory," in 2011 IEEE GLOBECOM Workshops (GC Wkshps), pp. 1199-1204, Dec 2011.

[17] J. Ma, X. Wang, and X. Lan, "Small-signal stability analysis of microgrid based on perturbation theory," in 2012 Asia-Pacific Power and Energy Engineering Conference, pp. 1-4, March 2012.

[18] B. S. Hartono, Y. Budiyanto, and R. Setiabudy, "Review of microgrid technology," in QiR (Quality in Research), 2013 International Conference on, pp. 127-132, June 2013.

[19] A. Parisio, E. Rikos, and L. Glielmo, "A model predictive control approach to microgrid operation optimization," Control Systems Tech nology, IEEE Transactions on, vol. 22, no. 5, pp. 1813-1827, 2014.

[20] P. C. Sekhar, S. Mishra, and R. Sharma, "Data analytics based neurofuzzy controller for diesel-photovoltaic hybrid ac microgrid," IET Generation, Transmission Distribution, vol. 9, no. 2, pp. 193-207, 2015.

[21] A. Bidram and A. Davoudi, "Hierarchical structure of microgrids control system," Smart Grid, IEEE Transactions on, vol. 3, no. 4, pp. 1963 1976, 2012.

[22] M. Savaghebi, A. Jalilian, J. C. Vasquez, and J. M. Guerrero, "Secondary control scheme for voltage unbalance compensation in an islanded droop-controlled microgrid," Smart Grid, IEEE Transactions on, vol. 3, no. 2, pp. 797-807, 2012.

[23] B. Milosevic and M. Begović, "Voltage-stability protection and control using a wide-area network of phasor measurements," Power Systems, IEEE Transactions on, vol. 18, no. 1, pp. 121-127, 2003.

[24] U. Orhan, M. Hekim, and M. Ozer, "EEG signals classification using the K-means clustering and a multilayer perceptron neural network model," Expert Systems with Applications, vol. 38, no. 10, pp. 13475-13481, 2011. 